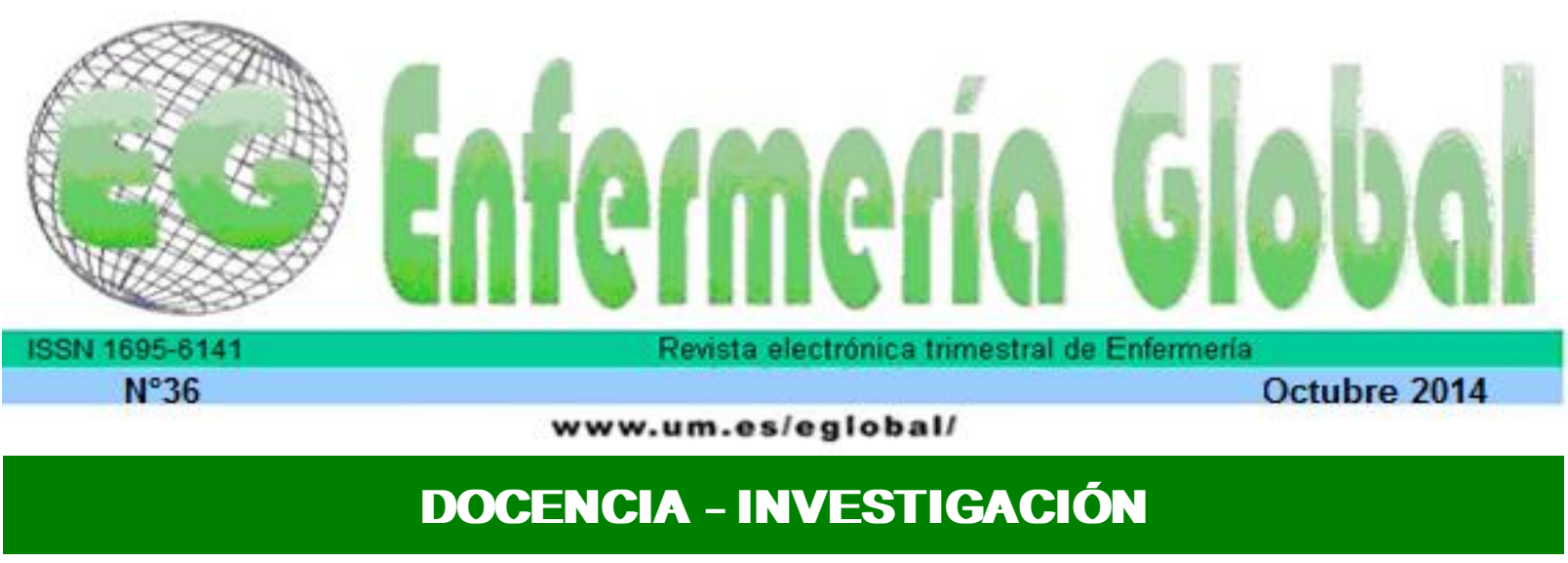

\title{
El uso de psicofármacos en individuos con trastorno mental en seguimiento ambulatorio
}

A utilização de psicofármacos em indivíduos com transtorno mental em acompanhamento ambulatorial

The usage of psychotropic drugs in subjects with mental disorder in ambulatory attendance

*Xavier, Mariane da Silva ${ }^{* *}$ Terra, Marlene Gomes ${ }^{* * *}$ da Silva, Cristiane Trivisiol *Souto, Valquiria Toledo **Mostradeiro, Sadja Cristina Tassinari de Souza ****Vasconcelos, Raíssa Ottes

\begin{abstract}
*Enfermera. Alumna dee Máster del Programa de Postgraduación en Enfermería de la Universidad Federal de Santa Maria (UFSM). E-mail: marianesxavier@yahoo.com.br ** Doctora en Enfermería. Profesora del Departamento de Enfermería y Postgraduación de la UFSM. ***Máster en Enfermería. Doctoranda del Programa de Postgraduación en Enfermería de la Universidad Federal de Rio Grande do Sul (UFRGS) ${ }^{* * * * E s t u d i a n t e ~ d e ~ E n f e r m e r i ́ a ~ d e ~ l a ~ U n i v e r s i d a d ~ F e d e r a l ~ d e ~ S a n t a ~ M a r i a . ~ B o l s i s t a ~}$ FIPE. Brasil.
\end{abstract}

Palabras clave: Enfermería; Salud mental; Psicotrópicos; Atención ambulatoria; Análisis cualitativo

Palavras chave: Enfermagem; Saúde mental; Psicotrópicos; Assistência ambulatorial; Análise qualitativa.

Keywords: Nursing; Mental health; Psychotropic drugs; Ambulatory care; Qualitative analysis

\section{RESUMEN}

Objetivo: Conocer las experiencias de las personas con enfermedad mental en el uso de psicofármacos.

Metodología: Investigación cualitativa realizada mediante entrevista semiestructurada a 15 participantes, entre abril y mayo de 2012, en un hospital universitario del interior del estado. Los resultados fueron procesados por el software de Atlas TI y sometidos a análisis de contenido temático.

Resultados: Las experiencias involucran aspectos que motivan o limitan el uso de drogas psicotrópicas. Como aspectos motivadores se identificó la percepción de los usuarios de los beneficios que las drogas psicotrópicas pueden causar, por ejemplo, la reinserción social y la mejora de la vida familiar. Del mismo modo, las dificultades en el uso de drogas psicotrópicas estaban relacionadas con el conocimiento de los efectos secundarios que se pueden presentar. 
Conclusión: Se concluye que para las personas con enfermedades mentales las drogas psiquiátricas ayudan en el tratamiento, a pesar de sus reacciones adversas. Al mismo tiempo, esto no debe ser visto como la única forma de tratamiento, pero debe combinarse con asesoramiento psicosocial, grupo familiar, atención multidisciplinar, es decir, debemos ofrecer un cuidado cualificado, atención integral y de acuerdo a la necesidad de cada individuo.

\section{RESUMO}

Objetivo: Conhecer as experiências de indivíduos com transtorno mental frente à utilização de psicofármacos.

Metodologia: Pesquisa qualitativa, realizada por meio de entrevista semiestruturada com 15 participantes, no período de abril a maio de 2012, em um hospital de ensino do interior do estado. Os achados foram processados pelo software Atlas $\mathrm{TI}$ e, submetidos à análise de conteúdo temática.

Resultados: As experiências envolvem aspectos que motivam ou dificultam a utilização de psicofármacos. Como aspectos motivadores identificou-se a percepção do usuário acerca dos benefícios que os psicofármacos podem gerar, como a reinserção social e melhora no convívio familiar. Da mesma forma, as dificuldades para o uso dos psicofármacos foram relacionadas com a consciência acerca das reações adversas que estes podem apresentar.

Conclusão: Conclui-se que para indivíduos com transtorno mental os psicofármacos auxiliam no tratamento, apesar de suas reações adversas. Ao mesmo tempo este não deve ser visto como a única forma de tratamento, mas sim deve ser aliado a acompanhamento psicossocial, grupo de familiares, atenção multiprofissional, ou seja, deve-se ofertar um cuidado qualificado, integral e de acordo com a necessidade de cada indivíduo.

\section{ABSTRACT}

Objective: Knowing the experiences from subjects with mental disorder face to the usage of psychotropic drugs.

Methodology: Qualitative research conducted by semi-structured interviews with 15 participating, in a period from April to May 2012, in a teaching hospital from countryside. The results were processed by the Atlas TI software and were submitted to analysis of thematic content.

Results: The experiments involved different aspects, the ones that motivate or hinder the use of psychotropic drugs. The perception of the users concerning the benefits that psychotropic drugs can bring was identified as a motivated aspect, because it provides the social reintegration and the improvement on living together. Similarly, the difficulties to the use of psychotropic drugs were related to the conscience regarding the adverse reactions that these drugs may present.

Conclusion: It was conclude that to the subjects with mental disorder the psychotropic drugs are efficient for the treatment, despite its adverse reactions. At the same time, the psychotropic drugs must not being seen as the only way of treatment, but they must be allied to the psychosocial attendance, family group, multi-professional attention, in other words, it must be offered a full and qualified care according to the necessity of each subject.

\section{INTRODUCCIÓN}

De acuerdo con el Consejo Nacional de Salud (CNS), los trastornos mentais afectan a cerca del $12 \%$ de la población brasileña, lo que corresponde a 23 millones de personas que necesitan de alguna atención en salud mental, sea de forma continua o eventual ${ }^{(1)}$. Esta atención demanda acciones multiprofesionales en la esfera psicosocial, como la realización de talleres terapéuticas, grupos de convivencia, visita domiciliaria, consulta de enfermería, psicoterapia y demás terapias individuales y colectivas, que sean capaces de invertir en el potencial de salud del individuo con sufrimiento psíquico ${ }^{(2)}$. Además de estas, se destaca como parte de las acciones terapêuticas, el tratamiento medicamentoso. 
La terapéutica medicamentosa para el tratamiento en salud mental se da por el uso de psicofármacos, que alteran la actividad psíquica aliviando los síntomas de los trastornos psiquiátricos ${ }^{(3-2)}$. Sus efectos sufren influencias de diversos factores, como características individuales, estados patológicos y patrón de uso. Este tipo de tratamiento tuvo su inicio al final de la década de los 50, con la introducción de los neurolépticos $^{(2)}$. Los neurolépticos 0 antipsicóticos son psicofármacos inhibidores de las funciones psicomotoras que atenúan, también, los síntomas neuropsíquicos considerados psicóticos, tales como los delirios y alucinaciones ${ }^{(4)}$.

El descubrimiento de estos neurolépticos, en los años 50, no acabó, sin embargo, con el número de recaídas e ingresos, en lo que se refiere al tratamiento de las enfermedades mentales, contrariando las esperanzas de beneficios permanentes y estables. Se nota que después de la mejora de la sintomatología, los pacientes tienden a hacer uso irregular de sus medicinas, llegando incluso a interrumpir su tratamiento. Un posible factor para ese uso irregular es el tiempo de tratamiento.

Con el paso del tiempo, los pacientes pueden presentar un comportamiento desmotivado para el uso continuo y regular de la medicación. Así, una estrategia para mantener su uso es promover un cuidado que acerque al paciente a su cotidiano, con la finalidad de reordenar sus capacidades psicosociales y sus potencialidades en el curso de la enfermedad. Por este motivo, la rehabilitación psicosocial posee gran valía, pero para ello el tratamiento psicosocial que tiene por objetivo ayudar a los pacientes y familiares, necesita estar en sintonía con el tratamiento psicofarmacológico ${ }^{(2)}$.

En ese sentido, se entiende que los psicofármacos necesitan ser encarados como una de las posibilidades de tratamiento, y no la única, y que tendrán mayor éxito terapéutico si están asociados a la atención psicosocial, escucha cualificada, actividades resocializadoras, religiosidad, entre otras formas de asistencia que contemplen la integralidad del ser humano, según preconiza la Reforma Psiquiátrica.

La Ley no 10.216, Ley de la Reforma Psiquiátrica, reorienta el modelo de atención a la persona con trastorno mental. Este pasa de un modelo pautado en la lógica manicomial, a un modelo de atención psicosocial, el cual asegura al paciente entre otras cosas, una atención integral y ampliada, así como el derecho de ser parte activa en su tratamiento ${ }^{(5-6)}$.

En detrimento de lo que la referida ley prevé, se percibe que, en el cotidiano de los servicios de salud mental, la participación de los individuos en su tratamiento todavía se muestra de forma pasiva, la asistencia es verticalizada, no posibilita la autonomía y corresponsabilización de los individuos. Así, se observa que la asistencia prestada todavía está lejos de la ideal, carece de ser críticamente reflexionada antes de consolidarse.

Una estrategia para la consolidación de una asistencia cualificada en salud mental puede ser la ampliación de la relación interpersonal en el mantenimiento del tratamiento psiquiátrico, a fin de promover una mejor relación del paciente con su cuerpo, su círculo social, su familia y sus capacidades. Para la enfermería, profesión característicamente interactiva y sistemática durante el cuidado, este es el recorrido necesario para la resolución de problemas y manutención del tratamiento, respetando la integralidad, autonomía y libertad de sus pacientes ${ }^{(2) .}$ 
Todavía se cree que otra estrategia para la cualificación de la asistencia prestada sea la realización de estudios en el área de enfermería que tengan como tema la utilización de psicofármacos. Nos parece importante reflexionar sobre el espacio, el intersticio entre una investigación y el respectivo impacto en la atención prestada, especialmente, en el Sistema Único de Salud, ya que defendemos la asistencia de calidad en la salud como derecho inalienable de todo ciudadano, y no como mercadería que puede lograrse conforme la posición social y económica de cada sujeto en el proceso de producción ${ }^{(7)}$.

Corrobora esto un estudio bibliográfico realizado anteriormente, por medio de una búsqueda en las bases de dados Literatura Latino-Americana y del Caribe en Ciencias de la Salud (Lilacs) y Medical Literature Analysis and Retrieval System Online (Medline), utilizando las palabras clave "salud mental" y "psicofármacos", se evidenció escasez en cuanto a la producción de conocimiento acerca de la temática utilización de psicofármacos, principalmente, en investigaciones que utilizasen abordaje cualitativo y de autoría de enfermeros. En ese sentido, se justifica la necesidad de estudios que permitan comprender los significados que involucran al sujeto y el uso de psicofármacos en diferentes escenarios y aspectos de la salud mental.

Conocer las experiencias que permean la utilización de psicofármacos por individuos con trastorno mental instiga una reflexión a los profesionales de salud que actúan en los servicios, y posibilita fomentar una asistencia cualificada e integral en la atención a la salud mental de los usuarios.

En esta perspectiva, la cuestión orientadora de ese estudio fue: ¿cómo los individuos con trastorno mental experimentan la utilización de psicofármacos? Y, como objetivo: conocer las experiencias de individuos con trastorno mental frente a la utilización de psicofármacos.

\section{MATERIAL Y MÉTODO}

Esta es una investigación de tipo exploratoria y descriptiva con abordaje cualitativo, realizada con 15 individuos con trastorno mental, que frecuentaban el ambulatorio del servicio de psiquiatría de un hospital en el interior del estado del Rio Grande del Sur, en el periodo entre abril y mayo de 2012. La elección de los participantes ocurrió de manera intencional de modo que el número total fue definido por saturación de los datos, o sea, cuando las informaciones comenzaron a repetirse en la entrevista y los objetivos del estudio habían sido alcanzados ${ }^{(8-9)}$.

Los participantes fueron entrevistados en una sala del ambulatorio, en ambiente privado, en fecha y horario previamente agendados, por medio de una entrevista semiestructurada con la siguiente cuestión desenadenante: para usted, ¿cómo es utilizar una medicación psiquiátrica (psicofármacos)?

Fueron utilizados como criterios de inclusión: individuos adultos (hombres y mujeres) frecuentadores del ambulatorio, con enfermedades estables, que hicieran uso de psicofármacos. Y, como criterios de exclusión: aquellos individuos que presentasen confusión mental, limitaciones cognitivas o secuelas neurológicas, previamente diagnosticadas por su médico asistente, que limitaran la comprensión de los objetivos y cuestionamientos del estudio. 
Después de la realización de las entrevistas fueron transcritas integralmente y organizadas por medio del Software Atlas Ti 6.2 (Qualitative Research and Solutions). Primero, las declaraciones fueron leídas integralmente, después se realizó la codificación, extrayendo los temas que representaran el sentido de cada declaración. A partir de esos temas, se realizó la recodificación, donde los temas convergentes formaron las categorías temáticas ${ }^{(10)}$.

El estudio tuvo en consideración los principios éticos de la investigación involucrando seres humanos recomendados por la Resolución no 466/2012 del Consejo Nacional de la Salud ${ }^{(11)}$. El Protocolo del Proyecto de Investigación fue aprobado por el Comité de Ética en Investigación (CEP) de la Universidad Federal de Santa Maria (UFSM), bajo el no 00762612.0.0000.5346. Todos los participantes firmaron el Término de Consentimiento Libre y Aclarado.

\section{RESULTADOS}

Los resultados serán descritos, de acuerdo con la clasificación en dos categorías, que son: Usando Psicofármacos: posibilidades de (re)inserción familiar/social; y Reacciones adversas: del extrañamiento a la adaptación.

\section{- Usando Psicofármacos: posibilidades de (re)inserción familiar/social}

Los individuos con trastorno mental perciben que la utilización de psicofármacos les proporciona nuevas posibilidades de reinserción, tanto social como familiar.

Les gustaba cuando yo tomaba medicina, mi hermana siempre me mandaba tomar la medicina.(U3)

Al comienzo yo perdí muchos amigos, pierden la confianza en nosotros, creen que vamos a agredirlos, pero con el tiempo eso fue cambiando $y$ ahora ya estoy casi $100 \%$ volviendo con las amistades, eso se debe al tratamiento (medicamentos). (U14)

Los resultados también muestran que los individuos con trastorno mental demostraron aparente consciencia del sufrimiento causado a sus familiares, y perciben que el uso de las medicinas contribuye a la reestructuración familiar.

Ahora, eso ahí (trastorno mental) desestructuró toda mi familia, fue horrible! Ellos sufren y yo sufro también. Ellos sufren conmigo, yo sé. (U5)

Esa relación que yo tenía con la sociedad, de querer pelear, en casa que surgía. ;Yo estaba siempre rígido! Como estoy tomando las medicinas, yo no tengo voluntad de agresión. (U4)

Los individuos sienten la necesidad de aceptación delante de la sociedad, pero si tiene trastornos mentales está condicionado a vivir sin defensa, expuesto, juzgado y excluido.

Tú te pones una armadura blindada para armarte, para defenderte de los enemigos, solo que, sin embargo, tu enemigo es aquél que estás creando dentro de la armadura, dentro de tu cuerpo. (U4) 
Las personas van a juzgarte, van mirarte con una mirada distinta, tú vas a perder tus amistades, porque yo perdí mis amistades, „Perdí todo! (U5)

En medio a los conflictos personales vividos por los individuos que poseen trastornos mentales, muchas veces, estos pueden acabar encontrando refugio y esperanza en el tratamiento medicamentoso.

¡No basta la fe! La obra sería el tipo tomar la medicina, comprar la medicación, tomar la medicina en el horario exacto de la receta. Esa es la obra en nuestra vida y lo que va a cambiar. (U9)

Los resultados que compusieron esta categoría permiten inferir que el significado de la utilización de los psicofármacos para esos individuos pasa por las posibilidades que su uso, y la consecuente estabilización de cuadros psiquiátricos, pueden proporcionarles, como la posibilidad de reinserción familiar/social, de mejor relación familiar, y de aceptación

- Reacciones adversas: del extrañamiento a la adaptación

Para los participantes de este estudio las reacciones adversas presentaron importancia significativa, pues estuvieron presentes en la mayor parte de las declaraciones representando un aspecto que causa extrañamiento y dificulta la utilización de los psicofármacos. Las reacciones relatadas están relacionadas con la sedación, como disminución de la coordinación psicomotora, rigidez muscular y temblores.

Ese (nombre de una medicina antipsicótica) me dejó todo retorcido y mi padre aplicó una inyección de (nombre de una medicina anticolinérgica) y yo volví a la normalidad. (U1)

Al inicio quedaba duro, caminaba inclinado hacia frente, pero era efecto de la medicina. Otra dificultad que encontré con la medicina fue que me puse trémulo. (U3)

Encontré dificultad, ime quedaba dura! No podía hacer nada, quedaba toda dura. (U6)

Otras reacciones adversas descritas fueron el dolor epigástrico y la somnolencia excesiva.

Sentía una cosa incómoda en el cuerpo, no dejaba parar sentado. (U3)

Ella tiene el efecto secundario que es el sueño. (U4)

Me quedé dos días durmiendo, durmiendo sin parar, sin que nadie me despertara. Tuve que quedarme durmiendo, no resolvió. (U5)

Es medio difícil porque la medicina tiene el lado bueno y el malo, que perjudica el estómago. (U7) 
Las reacciones adversas de los psicofármacos también son vistas por los participantes del estudio como algo momentáneo, pues después de algún tiempo de tratamiento se sienten bien.

Yo tomo la medicina correctamente. Me daba vértigo, pero ahora estoy bien. (U13)

En los primeros tiempos me incomodaba, me daba malestar, no había condiciones de hablar, pero después, con el tiempo, vamos adaptándonos con la medicina,jtodo mejoró! No 100\%, pero alrededor de $70 \%$ mejoró. (U14)

Los individuos, al adherir al tratamiento medicamentoso prescrito, tienen que convivir con reacciones adversas, lo que, a veces, limita la espontaneidad de sus acciones cotidianas. Por otro lado, si abandonan el tratamiento, temprano o tarde viven la crisis que además de limitar sus acciones, puede exponerles a situaciones vejatorias, ocasionando pérdidas importantes, afectivas, sociales, entre otras ${ }^{(12)}$.

\section{DISCUSIÓN}

Las informaciones resultantes de este estudio reafirman que ser un individuo con trastorno mental en una sociedad que camina lentamente para la inclusión de los "diferentes" consiste en una experiencia permanentemente desafiadora. Para los sujetos de ese estudio el uso de psicofármacos posibilita una mejor relación en el ambiente familiar, siendo reconocido el estímulo de la familia para el mantenimiento del uso.

Además de eso, el uso de los psicofármacos fue visto como una posibilidad de restablecimientos de la confianza y de los lazos de amistad, una vez que su utilización los torna menos agresivos. En ese sentido, los individuos con trastorno mental reconocen que la aprobación de la familia, de los amigos y de la sociedad está condicionada a la utilización correcta de sus medicaciones y puede ser un estímulo para que este mantenga su tratamiento.

Se entiende que la familia ejerce papel fundamental junto al paciente cuando incentiva y colabora para que este haga el uso correcto de la medicación. No obstante, es importante subrayar que la convivencia con un familiar que posee trastornos mentales representa elevada sobrecarga para los familiares, dado que la enfermedad afecta no sólo al paciente, sino a toda la familia ${ }^{(13-14)}$.

La necesidad de acordar o incentivar a los individuos con trastornos mentales a volver a tomar sus medicamentos, la convivencia con alteraciones bruscas de humor, vivir en constante vigilancia para prevenir riesgos, y la vulnerabilidad a las situaciones de agresividad, entre otras situaciones, son factores que permean la relación con el individuo con trastorno mental, contribuyendo a desestructurar las relaciones familiares $^{(15)}$.

Corroborando lo expuesto, las repercusiones del trastorno mental para la familia fueron señaladas por los sujetos de ese estudio como causadoras de sufrimiento tanto para el individuo como para su familiar, estando relacionado con la desestructuración familiar. Para esos individuos, esas repercusiones, ejemplificadas por la agresividad, serian amenizadas por el uso de medicación. 
Los trastornos mentales crean una barrera que dificulta el contacto del individuo con el ambiente en que está inserto, alienándolo, a veces privándolo de su libertad y de la posibilidad de comvivir con los demás ${ }^{(16)}$. Al hacer una analogía con una armadura, el U4 percibe el trastorno mental como una barrera que, mientras protege, le impide convivir en sociedad. En ese contexto las concepciones del "loco" que culturalmente fueron incorporadas por la sociedad, como manera de juzgar, contribuyen a un escenario de prejuicio y exclusión social.

Otras declaraciones evidencian que el individuo demuestra conocer la necesidad de terapia medicamentosa y la ve como protagonista de su tratamiento, aunque haya dificultades inherentes a esta. Además de eso, la utilización de los medicamentos le da la oportunidad de transformar su vida, siendo considerada una verdadera "obra" que le permitirá tener una vida "normal".

Con todo, se cree que solamente ocurrirán transformaciones en la manera de convivir con el estigma de la enfermedad mental, posibilitando la reinserción de esos individuos, cuando haya un entendimiento global de la enfermedad y del cuidado, tanto del propio individuo, como de los familiares y profesionales de la salud. Más allá del tratamiento medicamentoso, se comprende que la asistencia necesita estar pautada en la lógica de atención psicosocial, la cual dirige sus acciones a la construcción de la ciudadanía, de la autoestima y de la interacción del individuo con la sociedad $^{(17)}$.

En ese sentido se percibe la importancia de la actuación de los profesionales de enfermería, pues, cuando presentan habilidades y actitudes para desarrollar acciones terapéuticas y educativas junto a los pacientes psiquiátricos, pueden contribuir efectivamente a la mejora de su condición biopsicosocial. Trabajar las dificultades del paciente en su relación consigo mismo y con los otros, promover el conocimiento sobre sus medicaciones, su plan terapéutico, y todo lo que involucra su tratamiento, de manera general, posibilita mayor autonomía al individuo, dándoles posibilidades para su reinserción social.

En relación con el tratamiento medicamentoso, el enfoque principal de las declaraciones reunidas en la segunda categoría de ese estudio está relacionado con las reacciones adversas de los psicofármacos. La Organización Mundial de la Salud (OMS) define reacción adversa a medicamento (RAM) como reacciones nocivas y no intencionales, que ocurren en dosis normalmente usadas por el ser humano para profilaxis, diagnóstico, terapia de la enfermedad o para la modificación de funciones fisiológicas ${ }^{(18)}$.

Los medicamentos que actúan sobre el sistema nervioso, como los psicofármacos, son utilizados por una grande parte de la población brasileña y están involucrados en la ocurrencia de varias reacciones adversas ${ }^{(19)}$. Datos evidencian que los fármacos antidepresivos, neurolépticos y antiepilépticos están involucrados en los principales registros de sospecha de reacciones adversas, causando, en su mayoría, hospitalización o prolongación de la hospitalización, invalidez y hasta riesgo de vida $^{(20)}$.

En las declaraciones, los individuos con trastorno mental mencionan un antisicótico típico (o tradicional) de alta potencia, con eficacia bien establecida en el combate de síntomas, como delirios y alucinaciones, pero que presenta efectos secundarios que 
potencialmente comprometen la adhesión de los pacientes a la terapéutica, como temblores, rigidez muscular, inquietud psicomotora y parkinsonismo ${ }^{(3)}$.

La rigidez muscular, síntoma más relatado, es una reacción adversa común, especialmente de los antipsicóticos típicos potentes. Puede surgir así que se instituye la terapéutica antipsicótica y persistir durante todo el tratamiento. Se manifiesta tanto en los miembros superiores como en los inferiores, incluso dificultando la marcha ${ }^{(21)}$. Considerando las reacciones que esos psicofármacos pueden generar, y la prevalencia de individuos que todavía lo utilizan, es fundamental que en la fase inicial del tratamiento, cuando las reacciones se manifiestan con mayor frecuencia, el paciente y su familia sean orientados con relación a los efectos y evolución esperados. El conocimiento es fundamental para incentivar a los pacientes, contribuyendo a su mejor adaptación y adhesión a la medicación ${ }^{(22)}$.

Otra reacción adversa referida por los usuarios fue el dolor epigástrico, esta es una sensación de ardor en la región del estómago que puede ocurrir en el inicio del tratamiento con algunas medicinas estabilizadoras de humor, acompañado o no por náuseas y vómitos, especialmente, con personas con historia de gastritis y úlcera péptica $^{(21)}$. Ya la somnolencia, reacción adversa también mencionada en este estudio, está relacionada con la alteración del patrón de sueño para más ${ }^{(3)}$.

Ante esas situaciones, los individuos con trastorno mental evidenciaron en las declaraciones que el tratamiento medicamentoso necesita de un periodo para su adaptación. Después de ese período ellos identifican que hay mejora.

De esa forma, todas esas reacciones son factores que pueden llevar al uso discontinuo de la medicación, Con todo, la percepción de que los beneficios a largo plazo pueden ser compensatorios lleva a esos individuos a persistir en el uso hasta la reducción o eliminación de los síntomas y adaptación a la medicación. Los psicofármacos, por minimizar y controlar los síntomas de los trastornos mentales contribuyen al proceso de rehabilitación psicosocial, ayudando a los individuos a convivir en sociedad, con sus familiares y amigos ${ }^{(22)}$

Se subraya que, en el área de la salud mental, es frecuente la prescripción de muchos medicamentos (polifarmacia), la interacción medicamentosa, el sufrimiento de los usuarios con efectos adversos, la no adhesión al tratamiento y el uso abusivo de los medicamentos. En virtud de eso, se reitera la necesidad de orientación de usuarios y familiares en cuanto a la acción de los medicamentos, sus efectos y forma de utilización. Son comunes en los servicios de salud mental los cuestionamientos en relación con la terapia medicamentosa, lo que resalta la importancia de rellenar ese hueco de desinformación para ampliar la efectividad de los tratamientos ${ }^{(23)}$.

El enfermero, como profesional de la salud que está más cerca y tiene mayor contacto con el paciente, podrá auxiliar con el control y ajuste de medicaciones, y buscar establecer una relación terapéutica que sobrepase el carácter ideológico y sea potencialmente transformadora de experiencias que generan sufrimientos al individuo con trastornos mentales.

\section{CONSIDERACIONES FINALES}

El estudio se caracterizó por algunas consideraciones relevantes sobre la descripción de los individuos con trastorno mental sobre sus experiencias frente a la utilización de 
psicofármacos. A partir de los resultados de esta investigación, los psicofármacos se muestran como factores que posibilitan la reinserción social y la convivencia familiar, a medida que su uso atenúa los síntomas de las enfermedades mentales, proporcionando así una mayor aceptación frente a una sociedad donde lo aceptable es la "persona normal".

En cuanto a la convivencia familiar, los individuos apuntan que sus familiares prefieren cuando estos utilizan correctamente la medicación, pues así se presentan más calmados. Como factor negativo señalan las reacciones adversas que algunos medicamentos presentan. Aún así, las declaraciones evidencian que los beneficios de la utilización del psicofármaco superan los efectos adversos, pues la adaptación a estos viene con el tiempo de uso.

Esta investigación proporcionó dar voz al individuo con trastorno mental que es sometido al tratamiento de psicofármacos, pues este pudo demostrar lo que eso implica en su vida. Para la enfermería, es relevante conocer las experiencias de individuos con trastorno mental frente a la utilización de psicofármacos para que pueda obtener un cuidado más integral y cualificado. Vale señalar que el uso de psicofármacos no debe ser visto como la única forma de tratamiento, pero debe estar aliado a otras estrategias, como grupos terapéuticos, atención multiprofesional, grupo de familiares, religiosidad, entre otros.

Por lo tanto, se cree que los resultados de ese estudio puedan facilitar discusiones entre los profesionales de salud y también la realización de nuevas investigaciones acerca de algunos principios en cuanto a la Reforma Psiquiátrica y sobre los sentidos de la integralidad en la atención al individuo con trastorno mental, enfatizando una práctica volcada para el usuario y sus necesidades que están de acuerdo con la realidad que cada uno trae consigo.

\section{REFERENCIAS}

1- Lourenço L. Transtornos mentais atingem 23 milhões de pessoas no Brasil. Agência Brasil [internet]. 2010 [acesso em 24 fev 2014]. Disponível em :http://memoria.ebc.com.br/agenciabrasil/noticia/2010-06-28/transtornos-mentaisatingem-23-milhoes-de-pessoas-no-brasil.

2- Cardoso L, Galera SAF. O cuidado em saúde mental na atualidade. Rev Esc Enferm USP [internet]. 2011 [acesso em 20 Abr 2012]45(3):687-91. Disponível: http://www.scielo.br/pdf/reeusp/v45n3/v45n3a20.pdf

3- Stefanelli MC, Fukuda IMK, Arantes EC. Enfermagem Psiquiátrica: Em Suas Dimensões Assistenciais. Manole LTDA, 2008.

4- Adela PE, Santiago GM, Laura PC, Ignacio GC. Psicofarmacología de la Psicosis: Elección del Fármaco, Adherencia al Tratamiento y Nuevos Horizontes. Clínica y Salud [internet]. 2010 [acesso em 17 Jan 2014]21(3): 271-283. Disponível em: http://scielo.isciii.es/scielo.php?script=sci arttext\&pid=S1130-

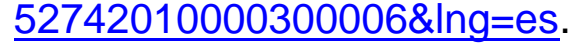

5- BRASIL. Lei n. 10.216, de 6 de abril de 2001. Dispõe sobre a proteção e os direitos das pessoas portadoras de transtornos mentais e redireciona o modelo assistencial em saúde mental. Presidência da República: Lei da Reforma Psiquiátrica. 2013. Disponível em: http://www.planalto.gov.br/ccivil 03/Leis/LEIS 2001/L10216.htm.

6- Amarante P. Loucos pela Vida: a trajetória da Reforma Psiquiátrica no Brasil. 2 edição. Rio de Janeiro: Fiocruz; 2013. 
7- Fortuna CM, Mishima SM. A pesquisa de enfermagem e a qualificação da assistência: algumas reflexões. Rev. Eletr. Enf. [internet]. 2012 [acesso em 15 Jan 2014]14(4):740-2.

Disponível

em:

http://www.fen.ufg.br/revista/v14/n4/pdf/v14n4a01.pdf.

8- Turato ER. Métodos qualitativos quantitativos na área da saúde: definições, diferenças e seus objetos de pesquisa. Rev Saúde Pública. [Internet] 2005; 39(3): 507-14 [acesso em 15 Jul 2013]. Disponível em: http://www.fps.usp.br/rsp>.15/10

9- . Minayo MCS. O desafio do conhecimento: pesquisa qualitativa em saúde. $8^{a}$ edição. São Paulo: Hucitec; 2010.

10- Mostardeiro SCTS, Rubim ENP. O cuidado de enfermagem em situações de alteração da imagem facial. Rev Gaúcha Enferm. [internet] 2011;32(2):294 301[acessado em 20 ago 2013]. Disponível em: http://seer.ufrgs.br/index.php/RevistaGauchadeEnfermagem/article/view/13970/12774. 11- BRASIL. Ministério da Saúde. Conselho Nacional de Saúde. Diretrizes e normas regulamentadoras sobre pesquisa envolvendo seres humanos. Resolução 466. 2012. 12- Miasso Al. "Entre a Cruz e a espada": o significado da terapêutica medicamentosa para a pessoa com transtorno afetivo bipolar, em sua perspectiva e na do familiar [tese]. Ribeirão Preto (SP). Universidade de São Paulo, 2006.

13- Albuquerque EPT, Cintra AMO, Bandeira M. Sobrecarga de familiares de pacientes psiquiátricos: comparação entre diferentes tipos de cuidadores. J Bras Psiquiatr. 2010;59(4):308-316.

14- Barbosa PL, Ramos OI, Cardoso GRI, Harter J. Consumo de crack: repercussões na estrutura e na dinâmica das relações familiares. Enfermería Global [internet] 2012; 25:150-160. [acesso em 20 fev 2014]. Disponível em: http://scielo.isciii.es/pdf/eg/v11n25/pt docencia3.pdf .

15- Filho MDS, Sousa AO, Parente ACBV, Martins MCC. Avaliação da sobrecarga em familiares cuidadores de pacientes esquizofrênicos adultos. Psicologia em Estudo [internet] 2010;15(3)639-647. [acesso em 16 jan 2014]. Disponível em: http://www.scielo.br/pdf/pe/v15n3/v15n3a22.pdf .

16- Almeida ACMCH, Felipes L, Dal Pozzo VC. O impacto causado pela doença mental na família. Revista Portuguesa de Enfermagem de Saúde Mental [internet] 2011; 6:40-47 [acesso em 29 Jan 2014]. Disponível em: http://www.scielo.gpeari.mctes.pt/scielo.php?script=sci arttext\&pid=S1647$21602011000200007 \& \operatorname{lng}=\mathrm{p}$.

17- Benevides DS. et al. Mental healthcare through therapeutic groups in a day hospital: the healthcare workers' point of view. Interface - Comunic. Saude, Educ. 2010;14(32):127-38.

18- OMS. La farmacovigilância: garantia de seguridade em el uso de los medicamentos. Perspectivas políticas de la OMS sobre medicamentos. 2004; 1-6 [acesso em 25 Mai 2012]. Disponível em: http://portal.anvisa.gov.br/wps/wcm/connect/c0201700474586a3901bd43fbc4c6735/R ACINE RAM.pdf?MOD=AJPERES.

19- Camargo AL, Ferreira MBC, Heineck I. Adverse drug reactions: a cohort study in internal medicine units at a university hospital. Eur J Clin Pharmacol. 2006;62:143-9.

20- Fonteles et al. Reações adversas causadas por fármacos que atuam no sistema nervoso: análise de registros de um centro de farmacovigilância do Brasil. Revista de Psiquiatria Clínica [internet] 2009; 36(4)[acesso em 25 Jan 2014]. Disponível em http://www.hcnet.usp.br/ipq/revista/vol36/n4/137.htm

21- Cardioli AV. Psicofármacos: consulta rápida. 4aa edição. Porto Alegre: Artimed; 2011. 
22- Carioni FS. O papel dos antipsicóticos no processo de reabilitação psicossocial do sujeito com diagnóstico de esquizofrenia [monografia]. Palhoça (SC): Universidade do Sul de Santa Catarina, 2006.

23- Coelho JC, Braga GAP. Roda de conversa sobre medicamentos: construindo significados para o uso racional com usuários de um serviço de saúde mental. Ministério da saúde. [acesso em 22 jun 2012]. Disponível: http://bvsms.saude.gov.br/bvs/premio medica/pdfs/.../juliana souza.pdf 